\title{
A Retrospective Review of Bone Health Screening of at-risk Children and Adolescents: A Single Center Experience
}

Chun-cheung Antony Fu ( $\nabla$ dr.a.ccfu@gmail.com )

Princess Margaret Hospital https://orcid.org/0000-0002-1491-0895

Wing Hang Luk

Princess Margaret Hospital

\section{Research article}

Keywords: adolescents, bone health, children, DXA, vitamin D

Posted Date: July 8th, 2020

DOI: https://doi.org/10.21203/rs.3.rs-40556/v1

License: (c) (i) This work is licensed under a Creative Commons Attribution 4.0 International License.

Read Full License 


\section{Abstract}

Background Bone health surveillance that includes laboratory tests and dual-energy $\mathrm{x}$-ray absorptiometry (DXA) for patients with conditions that predispose them to a higher risk of osteoporosis is recommended. This study aimed to review current practice of such surveillance in a local tertiary referral centre.

Methods Retrospective review of clinical data of patients who underwent DXA from 2013 to 2017 inclusive. Laboratory test results and presence of osteoporotic risk which was defined as bone mineral density (BMD) Z-score -2 SD or less were documented.

Results This review consisted of 112 patients, 58 boys and age ranged from 1.1 to 20.3 years. Most referrals for DXA came from the subspecialty of nephrology (56.3\%). Vitamin D status was rarely evaluated in this cohort of patients, only $17.9 \%$ of subjects had their vitamin D level checked. Overall, osteoporotic risk was demonstrated in $37 \%$ of the subjects. Thalassaemia, other haematological diseases like chronic ITP, osteogenesis imperfecta and inflammatory bowel disease accounted for majority of cases with high risk of osteoporosis.

Conclusion DXA is underutilized in this center. Bone health surveillance and protection should be strengthened.

\section{Background}

Osteoporosis is recognized as one of the major public health problems (1). It is not limited to adults, and accumulating evidence suggests that it may have its root in childhood (2). Notwithstanding the fact that genetic factors account for $60-80 \%$ contribution of peak bone mass variability $(2-3)$, the majority of vulnerable patients are those with osteoporosis secondary to disease processes including immobility (e.g. cerebral palsy, cord injury, Duchenne Muscular Dystrophy (DMD)), poor nutrition, chronic inflammatory conditions like systemic lupus erythematous (SLE), inflammatory bowel disease (IBD), Thalassemia, hormonal disorders, and those on long-term offending medical treatment (e.g. glucocorticoid and antiepileptics). Patients exposed to these negative bone regulators may fail to achieve the expected gain in bone mineral density (BMD) and hence are at risk of sustaining fractures. Nonetheless, osteoporosis could be asymptomatic even in the presence of fractures. Such under-recognition could have significant healthcare management and resource implications. It underscores the importance of regular bone health assessment in individuals with at-risk conditions.

Clinical practice guidelines recommend regular bone health surveillance by measuring serum calcium and vitamin $D$ together with dual-energy $x$-ray absorptiometry (DXA) in individuals with the abovementioned at-risk conditions (4-6). DXA is widely accepted as the gold standard non-invasive assessment tool of bone health owing to its safety, speed, precision and reliability (4). Its radiation dose is one-tenth that of a standard chest radiograph. In our hospital, DXA service has been made available since 2009 serving two thousand patients a year in the Kowloon West Cluster. In this review we aimed to examine the current practice of bone health surveillance in at-risk children and adolescents. 


\section{Method}

This was a retrospective review of paediatric patients who underwent DXA (Lunar iDXA) between the period 1 July 2012 and 30 June 2017 in a tertiary endocrine referral center at the Princess Margaret Hospital (PMH), Hong Kong. $\mathrm{PMH}$ is the only referral center serving paediatric populations in two districts, namely Kwai Tsing and Tsuen Wan of the Hong Kong territory. Population data obtained by the Hong Kong Census and Statistics Department in 2016 showed the combined population of the two districts was 802,159 , equivalent to $11.5 \%$ of the total population of Hong Kong.

The primary outcome was to examine the clinical characteristics of patients that undertook DXA during the study period, including the bone health surveillance performed prior to scanning. As a secondary outcome, we intended to estimate the incidence amongst the diagnosis groups that have high risk to develop osteoporosis. Patient data were extracted from the Clinical Data Analysis and Reporting System (CDARS), a database managed by the Hong Kong Hospital Authority. The only exclusion criterion was children under the age of 5 years at the time of scanning when the reference normative data of DXA at and below that age was not available. This study has obtained approval from the Institutional Review Board of Research Ethics Committee of Kowloon West Cluster.

DXA results were reported as BMD Z-score - the number of standard deviations (SD) of lumbar spine (LS) from the norm of the manufacturer's reference data. The International Society for Clinical Densitometry (ISCD) states the presence of a bone mineral content (BMC) or BMD Z-score of greater than or equal to 2 standard deviations below the reference values, evaluated by DXA of the lumbar spine indicates a reduced bone mass in children and adolescents and is associated with increased fracture risk. (5) The ISCD also recommends to report Z-score, rather than T-score, for skeletal health assessment in children with increased risk of fracture as many of them have not achieved their peak bone mass (6).

Demographic (age, gender and ethnicity), anthropometric parameters (body height, weight and BMI with corresponding Z-score), disease entity, laboratory (bone profile - serum calcium, phosphate and alkaline phosphatase, 25-hydroxyvitamin D (25OHD) and parathyroid hormone (PTH) levels) and treatment for osteoporosis if any were collected. For medication history, concurrent use of glucocorticoid and other offensive medications like anti-epileptics and immunosuppressants was also recorded.

\section{Results}

Over the 5-year study period, 112 subjects underwent DXA and the median age at the time of scanning was 15.5 years [IQR $11.4-17.6$ years] and $51.8 \%$ was male.

Figure 1 shows the referral pattern. Most referrals came from nephrology $(n=63)$, followed by endocrinology $(n=10)$, haematology $(n=10)$, rheumatology $(n=8)$, neurology $(n=7)$ and respirology $(n=$ 6). Overall, $37 \%$ of the patients had high risk of osteoporosis based on their DXA results. Table 1 summarizes the demographic and other basic information of the cohort. Bone profile (Calcium, phosphate and alanine phosphatase) was evaluated in $95.5 \%$, Vitamin D in $17.9 \%$ and PTH in $16.1 \%$ of 
the patients referred for DXA. While as per global guidelines, the lowest acceptable vitamin $D$ level is $50 \mathrm{nmol} / \mathrm{L}(10-11)$, the median level of vitamin $\mathrm{D}$ in this cohort was $48 \mathrm{nmol} / \mathrm{L} .85$ of them $(76 \%)$ were treated with calcium and vitamin D supplements (Cholecalciferol or Calcitriol). 73 of them $(65.2 \%)$ were on glucocorticoid (GC) therapy for 3 months or longer, and 70 of them (62.5\%) were on at least one immune-modulating therapy in addition to GC.

Table 2 shows subspecialty-based information for bone health management. The median age at the time of scanning was the greatest for patients referred from rheumatology, nephrology and hematology. The median BMD was most suboptimal in patients referred from gastroenterology (median BMD LS Z-score $2.8)$, followed by hematology (-2.7) and metabolic medicine (-2.1). $85.7 \%$ of nephrology and all rheumatology patients were prescribed glucocorticoid and immune-modulating therapy concurrently. All neurology patients received glucocorticoid therapy.

With regard to bone health surveillance, while bone profile monitoring was regarded as fundamental for all subspecialties, evaluation of vitamin D status was rarely performed in most subspecialties, except neurology and endocrinology (both $42.9 \%$ ), not to mention PTH was also poorly monitored for all subspecialties but gastroenterology and endocrinology (both 50\%). In nephrology, only around $10 \%$ of their patients had their vitamin D and PTH status checked, when they were the greatest stakeholder in referring patients for DXA.

Table 3 reveals disease-specific information. Headcount of each disease in the hospital, as per CDARS search over the study period is also listed in the table. The majority of Thalassemia patients $(85.7 \%)$ were in the range of osteoporotic BMD, followed by other haematological diseases (75\%), OI (50\%) and IBD $(50 \%)$. All in all, at least $20 \%$ of patients in any group had DXA showing LS BMD in osteoporotic range. Compared to the active headcount of each disease in the hospital over the study period, even though all Ol, $75 \%$ of DMD and $44.4 \%$ of lupus nephritis patients had DXA done, they were the minority $(23.2 \%)$ in the cohort. The majority $(76.8 \%)$ had $20 \%$ or less of patients in each disease group never had DXA done over the study period. In Table 3, there are 19 nephrology patients (30.2\%) that received Calcitriol as vitamin D supplement.

\section{Discussion}

In this cohort, 112 subjects of similar gender distribution were identified, $60 \%$ of which came from nephrology. Overall, $37 \%$ of them had osteoporotic range of BMD but this percentage varied from 10.3 to $100 \%$ amongst the subspecialties. Majority of them had bone profile screened but only a minority got vitamin D and PTH checked. Many of the patients referred for DXA had already been started on calcium and vitamin D supplements.

The diagnosis of childhood osteoprosis cannot be set based on the bone densitometric measurements alone as it requires the presence of a clinically significant fracture history. Furthermore, being a 2D measurement of BMD, DXA has its limitations. BMD of chronically ill children are often underestimated owing to the fact that they are often small built, so do their bone sizes (8). In addition, there has been no 
unified protocol for skeletal assessment as the clinical approach varies by specific diagnoses and expected clinical outcome. Nonetheless, it is still highly recommended as a bone health screening tool for a number of primary and secondary disorders that have been associated with low bone mass and risk of fracture (6), including Thalassemia (9), DMD (10) (11) and IBD (12), when patients may benefit from the results of densitometry.

The prevalence of some groups of our patients with osteoporotic BMD is comparable to the literature findings, namely nephrotic syndrome (13), SLE (14) and Thalassemia (15). This implies the actual number of patients that are truly osteoporotic, according to the ISCD criteria, is lower. For osteogenesis imperfecta, understandably some of the patients responded to bone-active treatment including bisphosphonate therapy as shown by improvement in their DXA. For DMD, the prevalence of osteoporotic range BMD is lower than reported (16), that is likely explained by small sample size.

All subjects in the cohort were regarded by their parent teams to be at risk of osteoporosis before they were referred for DXA. Albeit that, not necessarily they had serum 25-hydroxyvitamin D (250HD) level evaluated (17.9\% overall). Undoubtedly there shows inadequacy of $250 \mathrm{HD}$ screening in some subspecialties in the cohort. Patients from neurology and endocrinology might have done it better yet their headcount was small compared to other subspecialties like nephrology. Being a recognized risk factor of fracture (17), 250HD should be universally included in the bone health screening protocols.

Except OI and DMD, the median age of DXA scanning for all other disease groups are above 16 years. Together with the fact that over the 5-year study period, the number of active headcounts in most disease groups are greater than the cohort, it is postulated that many of the patients are deprived of the opportunity of bone health surveillance and some of them should have undergone the scan earlier. Meanwhile, some disease entities that are vulnerable to osteoporosis are absent in this cohort, e.g. patients with cerebral palsy, history of cancers, leukemia and severe asthma on high dose inhaled corticosteroids (18). This suggests the under-recognition of the importance of bone health management in certain subspecialties.

The two commonly available preparations of vitamin D supplements for prevention of osteoporosis are ergocalciferol (vitamin D2) and cholecalciferol (vitamin D3). Calcitriol is the most active metabolite of vitamin $D$ and can easily lead to hypercalcemia and/or hypercalciuria (19). Therefore, calcitriol is not the drug of choice for preventing osteoporosis yet it is still being prescribed by some subspecialty teams, e.g. alongside with the prescription of long-term glucocorticoid therapy. Thus, further education of the different vitamin $D$ preparations and sharing of experience is important in maintaining and optimizing bone health management of our patients. Paediatricians from different subspecialties may have their own judgement for arranging bone health management, including the timing of initiation and what to include in the management plan. It would be beneficial if endocrinologists could take the lead to coordinate amongst the parent teams to execute the bone health protection strategy in a more timely and complete fashion. 
This review of practice serves as a pilot study aiming to cover the most needed disease groups of the hospital but not every paediatric patient, since there is a vast scope of patients that deserve bone health screening. Therefore only patients that underwent DXA were recruited as it was believed this limited group of patients was already "at risk" in their paediatricians' mind. However, this review is also subject to a number of limitations. First, our hospital was lack of reference normative paediatric data forTBLH BMD measurement for DXA, that is also recommended by ISCD. But fortunately on the one hand vertebrae are rich in trabecular bone that is more metabolically active particularly in children, they are the most sensitive skeletal sites for BMD assessment; on the other hand, the measurement of LS and TBLH BMD are often synchronized. Second, as aforementioned, BMD of short children are underestimated owing to their smaller bone size (8). These should be adjusted for their absolute height or height age to eliminate the size-related artefacts (2), that has not been done in many places including our hospital. Third, BMC or BMD has been shown to have high specificity but low sensitivity for osteoporosis (20). Bone fragility is dependent on a multitude of factors in addition to bone mass. For example, a larger bone is resistant to fracture than a small bone, given both bones have the same BMC or BMD. Therefore, we should not solely rely on bone densitometry data to stratify patients' risk to develop osteoporosis.

In future work, longitudinal follow-up of the same group of subjects after the coordination by endocrinologists might be helpful to prove the improvement of implementation of bone health protection strategy in the hospital. It is also recommended to replicate the study with subjects identified by the specific disease groups instead of limiting to those that have undergone DXA in order to have a more complete picture of the situation.

\section{Conclusion}

To summarize, bone healthy protection strategy is not commonly executed. The utilization of DXA is restricted to several subspecialties and is lacking among the others. The understanding of risks of bone fragility and hence awareness of bone health surveillance in children and adolescents need to be strengthened among paediatricians. A comprehensive bone health management consensus for high risk disease groups, by optimization of modifable factors, should be convened or collaborated by a designated team e.g. the endocrinologists for children during their growth before the occurrence of fractures and to maximize adult bone health. We also look forward to the availability of normative heightadjusted DXA data for LS and TBLH BMD assessment for children.

\section{Abbreviations}

25OHD: 25-hydroxyvitamin D

BMC: bone mineral content

BMD: bone mineral density

DMD: Duchenne muscular dystrophy 
DXA: dual-energy X-ray absorptiometry

ISCD: International Society for Clinical Densitometry

LS: lumbar spine

TBLH: total body less head

\section{Declarations}

\section{Ethics approval and consent to participate}

This study was approved by the Institutional Review Board or Research Ethics Committee of Kowloon West Cluster, Hospital Authority, Hong Kong.

Consent to participate is not applicable as some of these patients would no longer be followed up in the paediatric units. However, as the study only involves reviewing clinical and laboratory results that were already documented in the clinical management system (CMS), no addition information or investigations will be required. In addition, all these data will be anonymised and patient identity will not be an issue.

\section{Consent for publication}

Not applicable.

\section{Availability of data and material}

The datasets used and/or analysed during the current study are available from the corresponding author on reasonable request.

\section{Competing interests}

The authors declare no conflict of interest that could be perceived as prejudicing the impartiality of the review study reported.

\section{Funding}

The authors have had no study sponsor in: (1) study design; (2) the collection, analysis, and interpretation of data; (3) the writing of the report; or (4) the decision to submit the paper for publication. We received no honorarium, grant, or other form of payment to produce the manuscript. 


\section{Authors' contributions}

WHL contributed the data and AF analyzed and interpreted the patient data and wrote the first draft of the manuscripts. All authors read and approved the final manuscript.

\section{Acknowledgements}

Not applicable.

\section{References}

1. Ray NF, Chan JK, Thamer M, Melton LJ. Medical expenditures for the treatment of osteoporotic fractures in the United States in 1995: report from the National Osteoporosis Foundation. J Bone Miner Res Off J Am Soc Bone Miner Res. 1997 Jan;12(1):24-35.

2. Levine MA. Assessing bone health in children and adolescents. Indian J Endocrinol Metab. 2012 Dec;16(Suppl 2):S205-12.

3. Brown LB, Streeten EA, Shuldiner AR, Almasy LA, Peyser PA, Mitchell BD. Assessment of sex-specific genetic and environmental effects on bone mineral density. Genet Epidemiol. 2004 Sep;27(2):15361.

4. Nanes MS, Kallen CB. Osteoporosis. Semin Nucl Med. 2014;44(6):439-50.

5. Shepherd JA, Schousboe JT, Broy SB, Engelke K, Leslie WD. Executive Summary of the 2015 ISCD Position Development Conference on Advanced Measures From DXA and QCT: Fracture Prediction Beyond BMD. J Clin Densitom Off J Int Soc Clin Densitom. 2015 Sep;18(3):274-86.

6. 2019 ISCD Official Positions - Pediatric - International Society for Clinical Densitometry (ISCD) [Internet]. [cited 2020 Mar 1]. Available from: https://www.iscd.org/official-positions/2019-iscdofficial-positions-pediatric/

7. Institute of Medicine (US) Committee to Review Dietary Reference Intakes for Vitamin D and Calcium. Dietary Reference Intakes for Calcium and Vitamin D [Internet]. Ross AC, Taylor CL, Yaktine AL, Del Valle HB, editors. Washington (DC): National Academies Press (US); 2011 [cited 2020 Mar 11]. (The National Academies Collection: Reports funded by National Institutes of Health). Available from: http://www.ncbi.nlm.nih.gov/books/NBK56070/

8. Schoenau E, Land C, Stabrey A, Remer T, Kroke A. The bone mass concept: problems in short stature. Eur J Endocrinol. 2004 Aug;151 Suppl 1:S87-91.

9. Cappellini MD, Cohen A, Porter J, Taher A, Viprakasit V, editors. Guidelines for the Management of Transfusion Dependent Thalassaemia (TDT) [Internet]. 3rd ed. Nicosia (CY): Thalassaemia International Federation; 2014 [cited 2017 Sep 12]. Available from:

http://www.ncbi.nlm.nih.gov/books/NBK269382/ 
10. Buckner JL, Bowden SA, Mahan JD. Optimizing Bone Health in Duchenne Muscular Dystrophy. Int J Endocrinol [Internet]. 2015 [cited 2020 Mar 7];2015. Available from: https://www.ncbi.nlm.nih.gov/pmc/articles/PMC4466394/

11. Birnkrant DJ, Bushby K, Bann CM, Alman BA, Apkon SD, Blackwell A, et al. Diagnosis and management of Duchenne muscular dystrophy, part 2: respiratory, cardiac, bone health, and orthopaedic management. Lancet Neurol. 2018;17(4):347-61.

12. Pappa H, Thayu M, Sylvester F, Leonard M, Zemel B, Gordon C. Skeletal health of children and adolescents with inflammatory bowel disease. J Pediatr Gastroenterol Nutr. 2011 Jul;53(1):11-25.

13. Gulati S, Godbole M, Singh U, Gulati K, Srivastava A. Are children with idiopathic nephrotic syndrome at risk for metabolic bone disease? Am J Kidney Dis Off J Natl Kidney Found. 2003 Jun;41(6):11639.

14. Compeyrot-Lacassagne S, Tyrrell PN, Atenafu E, Doria AS, Stephens D, Gilday D, et al. Prevalence and etiology of low bone mineral density in juvenile systemic lupus erythematosus. Arthritis Rheum. 2007 Jun;56(6):1966-73.

15. De Sanctis V, Soliman AT, Elsedfy H, Yassin M, Canatan D, Kilinc Y, et al. Osteoporosis in thalassemia major: an update and the I-CET 2013 recommendations for surveillance and treatment. Pediatr Endocrinol Rev PER. 2013 Dec;11(2):167-80.

16. Ward LM, Hadjiyannakis S, McMillan HJ, Noritz G, Weber DR. Bone Health and Osteoporosis Management of the Patient With Duchenne Muscular Dystrophy. Pediatrics. 2018 Oct 1;142(Supplement 2):S34-42.

17. Munns CF, Shaw N, Kiely M, Specker BL, Thacher TD, Ozono K, et al. Global Consensus Recommendations on Prevention and Management of Nutritional Rickets. J Clin Endocrinol Metab. 2016 Feb;101(2):394-415.

18. Bachrach LK, Levine MA, Cowell CT, Shaw NJ. Clinical Indications for the Use of DXA in Pediatrics. In: Sawyer AJ, Bachrach LK, Fung EB, editors. Bone Densitometry in Growing Patients: Guidelines for Clinical Practice [Internet]. Totowa, NJ: Humana Press; 2007 [cited 2020 Mar 11]. p. 59-72. (Current Clinical Practice). Available from: https://doi.org/10.1007/978-1-59745-211-3_4

19. Letavernier E, Daudon M. Vitamin D, Hypercalciuria and Kidney Stones. Nutrients [Internet]. 2018 Mar 17 [cited 2020 Mar 8];10(3). Available from:

https://www.ncbi.nlm.nih.gov/pmc/articles/PMC5872784/

20. Nayak S, Edwards DL, Saleh AA, Greenspan SL. Systematic review and meta-analysis of the performance of clinical risk assessment instruments for screening for osteoporosis or low bone density. Osteoporos Int J Establ Result Coop Eur Found Osteoporos Natl Osteoporos Found USA. 2015 May;26(5):1543-54.

\section{Tables}

Table 1. Demographic and other basic information of the cohort. 


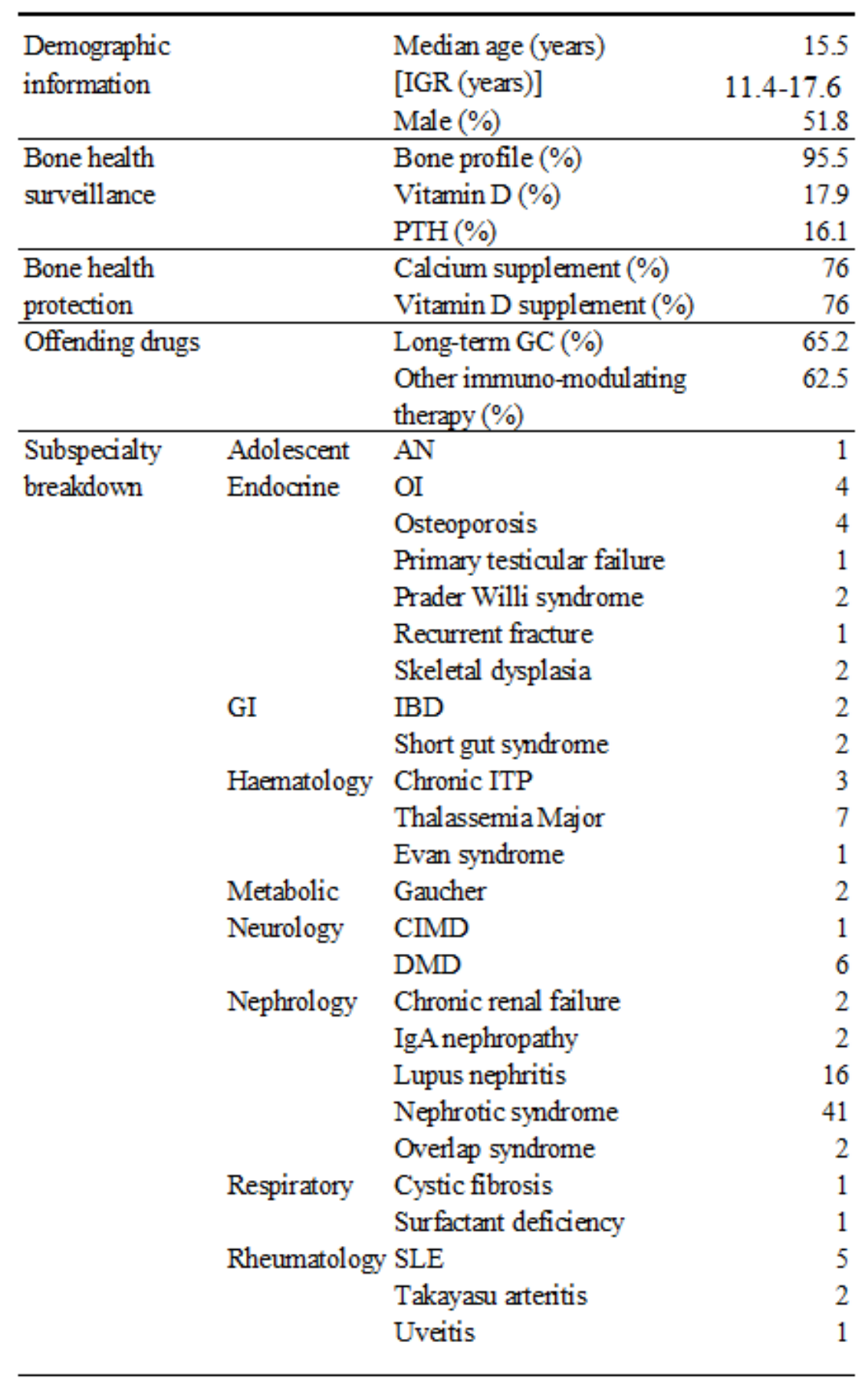

IGR: interquartile range; GC: glucocorticoid; AN: anorexia nervosa

OI: oste ogenesis imperfecta; GI: gastrointe stinal; IBD: inflammatory bowel dise ase

CIMD: chronic inflammatory demyelina ting disease; DMD: Duchenne Muscular Dystrophy

SLE: systemic lupus erythema tosis

Table 2. Subspecialty-based information for bone health surveillance and protection. 


\begin{tabular}{|c|c|c|c|c|c|c|c|c|c|c|c|c|c|c|c|c|c|c|c|c|}
\hline \multirow{2}{*}{ Subspecialty } & \multirow{2}{*}{$\begin{array}{r}\text { Headcount } \\
1\end{array}$} & Age (years) & Male, \% & $\begin{array}{l}\text { Median } \\
\text { Z-score }\end{array}$ & Bone health st & urveilla & $25 \mathrm{OHD}$ & & PTH & $\%$ & \multicolumn{6}{|c|}{$\begin{array}{l}\text { Bone health protection } \\
\text { Vitamin D supp }\end{array}$} & \multicolumn{4}{|c|}{ Offending medications } \\
\hline & & 18.6 & 0 & -0.6 & 1 & 100.0 & 0 & 0.0 & 0 & 0.0 & 1 & 100.0 & 1 & 100.0 & 0 & 0.0 & 0 & 0.0 & 0 & 0.0 \\
\hline Metabolic & 2 & 11.8 & 100 & -2.1 & 1 & 50.0 & 1 & 50.0 & 0 & 0.0 & 1 & 50.0 & 1 & 50.0 & 0 & 0.0 & 0 & 0.0 & 1 & 50.0 \\
\hline GI & 4 & 9.8 & 50 & -2.8 & 4 & 100.0 & 1 & 25.0 & 2 & 50.0 & 4 & 100.0 & 1 & 25.0 & 0 & 0.0 & 0 & 0.0 & 1 & 25.0 \\
\hline Respiratory & 6 & 5.3 & 50 & 1 & 2 & 100.0 & 2 & 100.0 & 1 & 50.0 & 1 & 50.0 & 2 & 100.0 & 0 & 0.0 & 0 & 0.0 & 1 & 50.0 \\
\hline Neurology & 7 & 9.6 & 100 & -1.6 & 7 & 100.0 & 3 & 42.9 & 0 & 0.0 & 5 & 71.4 & 6 & 85.7 & 0 & 0.0 & 7 & 100.0 & 7 & 100.0 \\
\hline Rheum atology & 8 & 15.4 & 25 & -2.05 & 8 & 100.0 & 0 & 0.0 & 0 & 0.0 & 8 & 100.0 & 8 & 100.0 & 0 & 0.0 & 8 & 100.0 & 8 & 100.0 \\
\hline Hem atology & 11 & 17.4 & 81.8 & -2.7 & 10 & 90.9 & 2 & 18.2 & 0 & 0.0 & 5 & 45.5 & 4 & 36.4 & 1 & 9.1 & 2 & 18.2 & 3 & 27.3 \\
\hline Endocrine & 14 & 10.4 & 78.6 & -1.2 & 10 & 71.4 & 6 & 42.9 & 7 & 50.0 & 4 & 28.6 & 4 & 28.6 & 1 & 7.1 & 0 & 0.0 & 0 & 0.0 \\
\hline Nephrology & 63 & 16.3 & 41.3 & -1.05 & 61 & 96.8 & 5 & 7.9 & 8 & 12.7 & 56 & 88.9 & 37 & 58.7 & 19 & 30.2 & 56 & 88.9 & 57 & 90.5 \\
\hline
\end{tabular}

Ca: calcium; PO4: phosphate; ALP. alanine phosphatase; 25OHD: 25-hydroxyvitamin D; PTH: parathyroid hormone; Ca supp: calcium supplement; GC: glucocorticoid therapy,

Others: immuno-modulating therapyother than GC

Table 3. Disease-specific information for bone health surveillance.

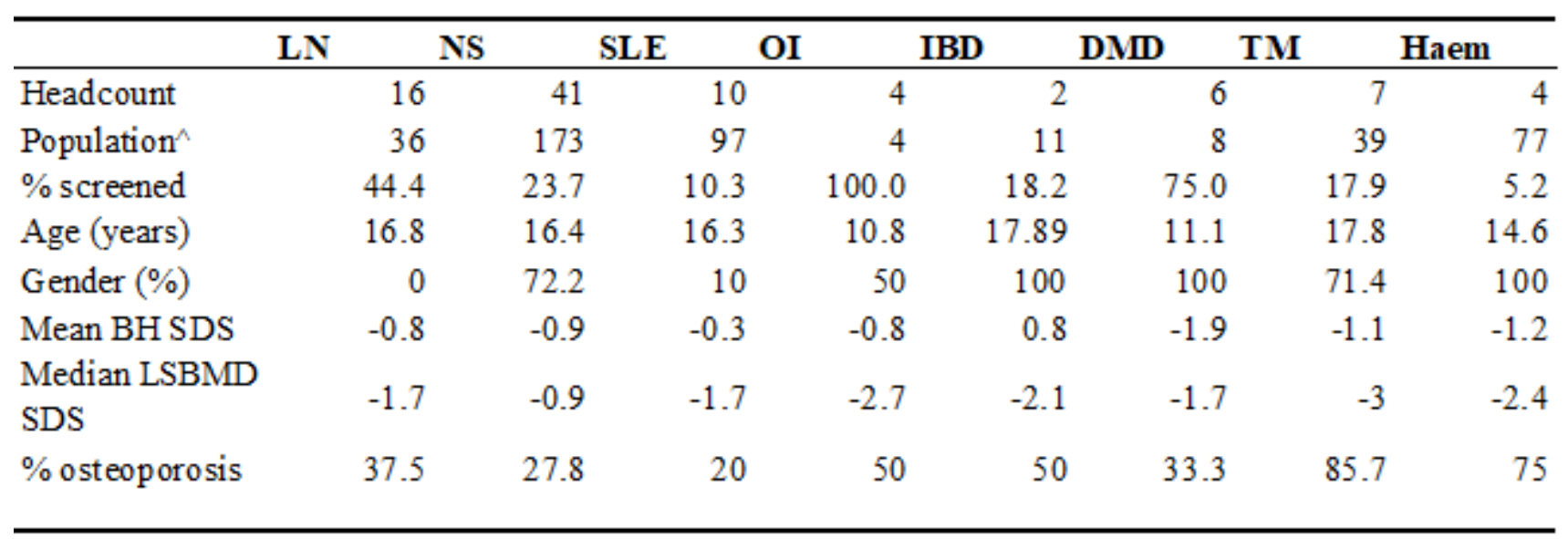

BH: body height, BW: body weight, LS: lumbar spine, BMD: bone mineral density

LN: hupus nephritis, NS: nephrotic syndrome, SLE: systemic lupus erythematosis, IBD: inflammatory bowel disease,

DMD: Duchenne Muscular Dystrophy, TM: Thalassemia Major, Haem: haematological diseases other than Cooley's anemia

'Refers to the active headcount of patients for each disease in PMH between 1 July 2013 and 30 June 2017 inchusively

\section{Figures}




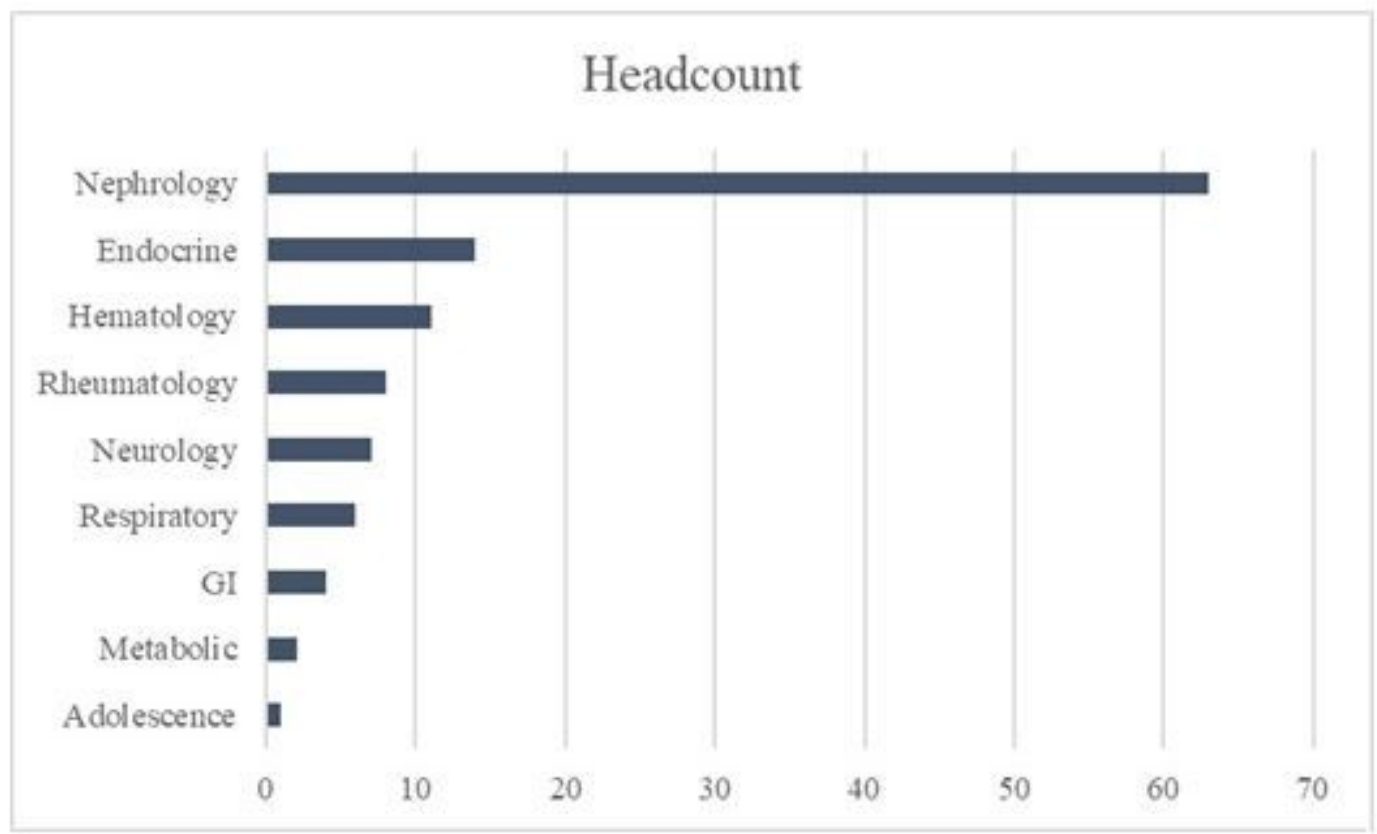

Figure 1

Referral pattern for DXA 